\title{
Erratum to: Practical Considerations for the Use of Daratumumab, a Novel CD38 Monoclonal Antibody, in Myeloma
}

Philippe Moreau $^{1}$ - Niels W. C. J. van de Donk ${ }^{2} \cdot$ Jesus San Miguel $^{3} \cdot$ Henk Lokhorst $^{2}$ • Hareth $\mathrm{Nahi}^{4} \cdot$ Dina Ben-Yehuda ${ }^{5} \cdot$ Michele Cavo $^{6} \cdot$ Gordon Cook $^{7} \cdot$ Michel Delforge ${ }^{8}$. Hermann Einsele ${ }^{9}$ Sonja Zweegman ${ }^{2} \cdot$ Heinz Ludwig ${ }^{10}$ - Christoph Driessen ${ }^{11}$. Antonio Palumbo $^{12}$. Thierry Facon ${ }^{13}$. Torben Plesner ${ }^{14} \cdot$ Meletios Dimopoulos $^{15}$. Pia Sondergeld ${ }^{16} \cdot$ Pieter Sonneveld $^{17} \cdot$ María-Victoria Mateos $^{18}$

Published online: 20 May 2016

(c) Springer International Publishing Switzerland 2016

\section{Erratum to: Drugs}

DOI 10.1007/s40265-016-0573-4

Table 4, which previously appeared as

Table 4 Management considerations concerning monoclonal antibody use in hematology in general, and myeloma in particular

\begin{tabular}{llllll}
\hline & \multicolumn{2}{l}{ CD38 monoclonal antibodies } & & \\
\cline { 2 - 6 } & Rituximab & Elotuzumab & Daratumumab & Isatuximab & MOR202 \\
\hline Infusion-related reactions & $\times$ & $\times$ & $\times$ & $\times$ & $\times$ \\
Interference with response assessment & $\times$ & $\times$ & $\times$ & $\times$ & $\times$ \\
Interference with blood typing & & & $\times$ & $\times$ \\
\hline
\end{tabular}

The online version of the original article can be found under doi:10.1007/s40265-016-0573-4.

Philippe Moreau

philippe.moreau@chu-nantes.fr

1 Hematology Department, University Hospital Hôtel-Dieu, Place Ricordeau, 44093 Nantes, France

2 VU University Medical Center, Amsterdam, The Netherlands

3 University Hospital Navarra, Pamplona, Spain

4 Karolinska University Hospital Huddinge, Stockholm, Sweden

5 Hadassah Medical Center, Jerusalem, Israel

6 University School of Medicine, Bologna, Italy

7 St James's University Hospital, Leeds, UK

8 Catholic University of Leuven, Leuven, Belgium
9 Julius Maximilian University, Würzburg, Germany

10 Wilhelminen hospital, Vienna, Austria

11 Kantonsspital St. Gallen, St. Gallen, Switzerland

12 University of Torino, Torino, Italy

13 University Hospital Lille, Lille, France

14 Vejle Hospital and University of Southern Denmark, Vejle, Denmark

15 University Athens School of Medicine, Athens, Greece

16 University of Giessen, Giessen, Germany

17 Erasmus MC Cancer Insitute, Rotterdam, The Netherlands

18 University Hospital of Salamanca, Salamanca, Spain 


\section{Should appear as}

Table 4 Management considerations concerning monoclonal antibody use in hematology in general, and myeloma in particular

\begin{tabular}{lccccc}
\hline & Rituximab & Elotuzumab & & \multicolumn{2}{c}{ CD38 monoclonal antibodies } \\
\cline { 5 - 6 } & & & Daratumumab & Isatuximab & MOR202 \\
\hline Infusion-related reactions & $\times$ & $\times$ & $\times$ & $\times$ & $\times$ \\
Interference with response assessment & $\times$ & $\times$ & $\times$ & $\times$ & $\times$ \\
Interference with blood typing & & & $\times$ & $\times$ \\
\hline
\end{tabular}

